Condition $\frac{\partial u}{\partial x}, \frac{\partial u}{\partial y}, \frac{\partial v}{\partial x}$, and $\frac{\partial v}{\partial y}$ all exist, so that, by the definition of a partial differential coefficient,

$$
\begin{gathered}
\frac{\partial u}{\partial x}+i \frac{\partial v}{\partial y}=\operatorname{Lt} \cdot \Delta_{1} w / \Delta_{1} z, \\
\frac{1}{i}\left(\frac{\partial u}{\partial y}+i \frac{\partial v}{\partial x}\right)=\operatorname{Lt} \cdot \Delta_{2} w / \Delta_{2} z
\end{gathered}
$$

which, using the Cauchy-Riemann equations, gives the equality of the unique. limits of $\Delta_{1} w / \Delta_{1} z$ and $\Delta_{2} w / \Delta_{2} z$, and the value of this limit given in the enunciation.

Again, as before, if $h k \neq 0$,

$$
\Lambda w=h \varepsilon_{1}(w)+i k \varepsilon_{2}(w),
$$

which shews, since the Secondary Condition is satisfied by $u$ and $v$, so that, if $h / k$ does not tend to zero, $\Delta w / h \rightarrow 0$, and, if $h / k \rightarrow 0$, $\Delta w / k \rightarrow 0$, - that, in either case, $\varepsilon_{1}(w)$ and $\varepsilon_{2}(w)$ both $\rightarrow 0$; therefore $\Delta w / \Delta z$ has the same unique limit as $\Delta_{1} w / \Delta_{2} z$ and $\Delta_{2} w / \Delta_{2} z$.

This proves the remaining half of the theorem.

\section{Une remarque sur les types de dimensions.}

Par

S. Ruziewicz (Lwów).

D'après M. Sierpiński ${ }^{1}$ ) le type de dimensions (au sens de M. Fréchet) $d H$ est dit le suivant pour le type $d E$ (et $d E$ le précédent pour $d H$ ), s'il n'existe ancun ensemble $Q$, tel que

$$
d E<d Q<d H \text {. }
$$

Pour abréger, nous écrirons dans ce cas

$$
d E \mathrm{p} d H .
$$

Le but de cette Note est de donner un exemple de cinq ensembles linéaires $P_{1}, P_{2}, P_{3}, Q_{1}$ et $Q_{2}$, tels que

\section{et}

$$
d P_{1} \mathrm{p} d P_{2} \mathrm{p} d P_{\mathbf{s}}
$$

$$
d P_{1} \mathrm{p} d Q_{1} \mathrm{p} d Q_{2} \mathrm{p} d P_{3}
$$

Cela prouvera que si le type de dimensions $d P_{2}$ est le suivant pour $d P_{1}$ et le précédent pour $d P_{8}, d P_{2}$ n'est pas nécessairement le type unique intermédiaire entre $d P_{1}$ et $d P_{3}$, et méme qu'il peut exister deux types intermédiaires entre $d P_{1}$ et $d P_{3}$.

Les ensembles $P_{1}, P_{2}, P_{3}, Q_{1}$ et $Q_{2}$ sont définis comme il suit.

$P_{1}$ est l'ensemble formé des nombres $0, \frac{1}{n}$ et $2+\frac{1}{n}(n=1,2,3, \ldots)$.

$P_{2}$ est formé des nombres 0 et $\frac{1}{2^{m}}+\frac{1}{2^{m+n}}(m=1,2, \ldots ; n=1,2, \ldots)$

$P_{s}$ est l'ensemble qu'on obtient en adjoignant à $P_{2}$ le point $\frac{1}{2}$.

1) Fund. Math. t. XIV, p. 122. 
$Q_{1}$ est l'ensemble qu'on obtient en adjoignant à $P_{1}$ le point 2.

$Q_{1}$ est formé des nombres $0,2, \frac{1}{n}, 2+\frac{1}{n}$ et $\left.4+{ }_{n}^{1}(n=1,2, \ldots)^{1}\right)$.

La démonstration que les cinq ensembles ainsi définis satisfont aux conditions imposées n'offre pas de difficultés.

1) M. Si erpiński désigne $Q_{1}$ par $H_{1}, P_{1}$ par $H_{2}$ et $P_{3}$ par $H_{3}$ ot ácrit (1. c., p. 123) que les types $d H_{1}$ et $d H_{2}$ sont des précédents pour lo type $d H_{3}$ : an lieu de $d H_{1}$ doit $\mathrm{y}$ être éridemment $d Q_{2}$.

\section{Über additive Massfunktionen in abstrakten Mengen.}

Von

\section{S tef a n B a a ch (Lwów).}

In einer vor kurzem gemeinsam mit Herrn Kuratowski veröffentlichten Note $\left.{ }^{1}\right)$ haben wir, unter Voraussetzung der Richtigkeit der Kontinuumhypothese $2 s_{0}=\boldsymbol{N}_{1}$, die folgende Verallgemeinerung eines bekannten Resultats von Herrn Vitali bewiesen:

Es gibt keine total additive nicht identisch verschwindende Mengenfunktion $m(X)$, welche jeder Teilmenge $X$ der Strecke $(0,1)$ eine reelle Zahl zuordnet und für alle aus nur einem Punkte bestehenden Mengen den Wert Null annimmt.

In diesem Satze kann die Strecke $(0,1)$ durch eine beliebige Menge von der Mächtigkeit des Kontinuums ersetzt werden. Die Funktion $m(X)$, welche wir wegen ihrer geometrischen Bedeutung eine Massfunktion, oder auch ein Mass von $X$ nennen, darf auch negative Werte annehmen.

In der vorliegenden Note beweisen wir einen allgemeineren, fur Mengen von beliebiger Măchtigkeit gültigen Satz, wobei wir die Richtigkeit der sog. Hypothese der Alephs, d. h. das Bestehen der Gleichung

$$
2 \mathrm{~s}_{\xi}=\mathrm{s}_{\xi+1}
$$

für beliebige Ordnungszahlen $\xi$ voraussetzen. Um diesen Satz aussprechen zu können, müssen wir zunăchst den Begriff der Massfunktion allgemein definieren.

Wir sagen, dass in einer Menge $E$ eine additive Massfunktion

1) S. Banach et C. Kuratowaki Sur une généralisation du probleme de la mesure Fund. Math. XIV (1929), pp. 127-131. 